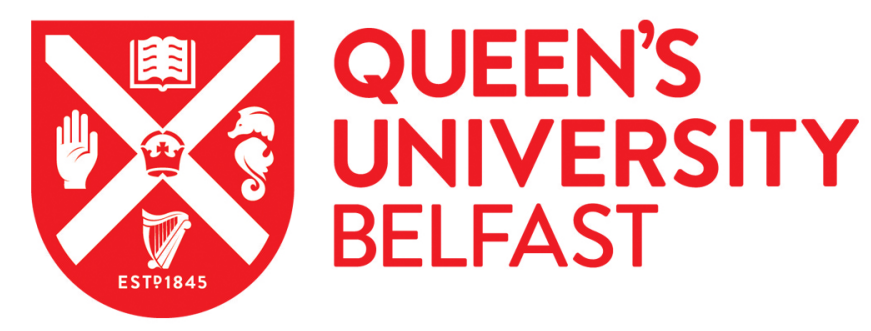

\title{
Investigation Into The Subambient Behavior Of Aqueous Mannitol Solutions Using Temperature-controlled Raman Microscopy
}

Beattie, J., Barrett, L., Malone, J., Nieuwenhuyzen, M., McGarvey, J., \& Kett, V. (2007). Investigation Into The Subambient Behavior Of Aqueous Mannitol Solutions Using Temperature-controlled Raman Microscopy. European Journal of Pharmaceutics and Biopharmaceutics, 67(2)(2), 569-578. https://doi.org/10.1016/j.ejpb.2007.03.015

Published in:

European Journal of Pharmaceutics and Biopharmaceutics

Document Version:

Early version, also known as pre-print

Queen's University Belfast - Research Portal:

Link to publication record in Queen's University Belfast Research Portal

\section{General rights}

Copyright for the publications made accessible via the Queen's University Belfast Research Portal is retained by the author(s) and / or other copyright owners and it is a condition of accessing these publications that users recognise and abide by the legal requirements associated with these rights.

Take down policy

The Research Portal is Queen's institutional repository that provides access to Queen's research output. Every effort has been made to ensure that content in the Research Portal does not infringe any person's rights, or applicable UK laws. If you discover content in the Research Portal that you believe breaches copyright or violates any law, please contact openaccess@qub.ac.uk. 


\author{
Research paper
}

\title{
Investigation into the subambient behavior of aqueous mannitol solutions using temperature-controlled Raman microscopy
}

\author{
J. Renwick Beattie ${ }^{\text {a }}$, Lindsay J. Barrett ${ }^{\text {a }}$, John F. Malone ${ }^{\text {b }}$, John J. McGarvey ${ }^{\text {a,b }}$, \\ M. Nieuwenhuyzen ${ }^{\mathrm{b}}$, Vicky L. Kett ${ }^{\mathrm{a}, \mathrm{c}, *}$ \\ a Centre for Clinical Raman Microscopy, The Queen's University of Belfast, Belfast, United Kingdom \\ b School of Chemistry and Chemical Engineering, The Queen's University of Belfast, Belfast, United Kingdom \\ ${ }^{c}$ The School of Pharmacy, The Queen's University of Belfast, Belfast, United Kingdom
}

Received 5 February 2007; accepted in revised form 21 March 2007

Available online 28 March 2007

\begin{abstract}
The aim was twofold; to demonstrate the ability of temperature-controlled Raman microscopy (TRM) to locate mannitol within a frozen system and determine its form; to investigate the annealing behavior of mannitol solutions at $-30{ }^{\circ} \mathrm{C}$. The different polymorphic forms of anhydrous mannitol as well as the hemihydrate and amorphous form were prepared and characterized using crystal or powder X-ray diffractometry (XRD) as appropriate and Raman microscopy. Mannitol solutions (3\% w/v) were cooled before annealing at $-30{ }^{\circ} \mathrm{C}$. TRM was used to map the frozen systems during annealing and was able to differentiate between the different forms of mannitol and revealed the location of both $\beta$ and $\delta$ polymorphic forms within the structure of the frozen material for the first time. TRM also confirmed that the crystalline mannitol is preferentially deposited at the edge of the frozen drop, forming a rim that thickens upon annealing. While there is no preference for one form initially, the study has revealed that the mannitol preferentially transforms to the $\beta$ form with time. TRM has enabled observation of spatially resolved behavior of mannitol during the annealing process for the first time. The technique has clear potential for studying other crystallization processes, with particular advantage for frozen systems.
\end{abstract}

(C) 2007 Elsevier B.V. All rights reserved.

Keywords: Amorphous; Polymorphism; Thermal analysis; Freeze-drying; Spectroscopy; Raman; Mannitol

\section{Introduction}

Mannitol is commonly included in pharmaceutical freeze-dried formulations since it confers a degree of robustness to the resultant products when it is allowed to crystallize. However, the subambient behavior of mannitol is significantly affected by the freeze-drying protocol as well as the presence of other species [1-5]. For example it has been shown that high protein concentrations can result in incomplete mannitol crystallization during freeze-drying, which may have serious consequences for the shelf stability

\footnotetext{
* Corresponding author. The School of Pharmacy, The Queen's University of Belfast, 97 Lisburn Road, Belfast BT9 7BL, United Kingdom. Tel.: +44 (0) 2890 972008; fax: +44 (0) 2890247794.

E-mail address: v.kett@qub.ac.uk (V.L. Kett).
}

of the product [6]. Much work has also been done to investigate the effect of common freeze-drying excipients on its behavior, e.g. lyoprotectants such as sucrose [7] and trehalose [8] which remain in the amorphous phase during freeze-drying as well as those such as glycine [6], sodium chloride [9] and buffers [10] which are known to crystallize and thus might be expected to promote the concomitant crystallization of the mannitol. Mannitol exhibits polymorphism $[1,11]$ and it is known that inclusion of excipients and the freeze-drying process affects the outcome of the crystallization process (for example [1,12]). There is also evidence that under certain freeze-drying conditions a metastable pseudopolymorph may be produced [13].

The uncontrolled crystallization of mannitol can cause problems such as vial breakage [14] therefore an annealing step is commonly included in the freeze-drying protocol 
when it is included in a formulation [15] in order to promote complete crystallization. However, mannitol-containing formulations can exhibit extended primary drying times after annealing, which is attributable to the crystalline material blocking pathways for release of vapor [16].

Previously [17], it was shown using modulated temperature differential scanning calorimetry (MTDSC), cold stage microscopy (CSM) and XRD that the amorphous mannitol formed on cooling 3\% (w/v) aqueous mannitol solutions crystallizes upon rewarming into the $\beta$ polymorph. CSM revealed that the crystallization process (detected with XRD and MTDSC at $-30^{\circ} \mathrm{C}$ [17]) correlated with the change in appearance of an excluded phase at the edge of a drop of mannitol solution. Annealing at $-30^{\circ} \mathrm{C}$ also appeared to promote further crystallization of the same form. In the current investigation the properties of $3 \%(\mathrm{w} / \mathrm{v})$ mannitol solutions were further examined using temperature-controlled Raman microscopy (TRM) with a view to determining how suitable the technique was for studying this system. Further objectives were to identify the process occurring at the edge of the drop as well as distinguishing into which polymorph the crystallization occurs.

Raman microscopy is one of a number of spectroscopic imaging techniques. Briefly, Raman spectroscopy uses a laser to irradiate a sample with monochromatic light. A small amount of this is scattered with a frequency shift, characteristic of the chemical bonds or molecules present in the material. This inelastic scattering of light gives rise to Raman spectra [18]. In TRM Raman spectroscopy is coupled with an optical microscope and a temperaturecontrolled stage. By carefully choosing the imaging conditions; laser; objective lens and confocal hole diameter, spatial and depth resolution can be controlled at the micron level. This therefore allows the investigation of the location of species within a sample and to observe how these materials behave as a function of temperature and/or time. The technique has been used to look at a range of pharmaceutical systems [19-22]. However, there are no published papers relating the use of the technique for the investigation of frozen systems.

\section{Materials and methods}

\subsection{Materials}

Mannitol was obtained from Roquette ("Pearlitol" Corby, UK; sorbitol $<0.9 \%$ mannose $0.02-1 \%$ )

Crystals of the $\alpha, \beta$, and $\gamma$ anhydrous polymorphs were prepared by the methods of Walter-Levy [23]. In order to produce mannitol hemihydrate the method of Nunes et al. [13] was used.

Mannitol solutions (3\% w/v) in distilled water were used in the sub-ambient studies. Aqueous solutions of mannitol were also prepared in the range $1-45 \% \mathrm{w} / \mathrm{v}$ in order to construct a Raman calibration curve at ambient temperature.

\subsection{Single crystal analysis}

\subsection{1. $X R D$}

Each of the three polymorphic forms of anhydrous mannitol was characterized not simply from unit cell parameters but from $a b$ initio single crystal diffraction data recorded on a Bruker SMART CCD diffractometer at ambient temperature, and subsequent structure analysis and refinement. The same single crystal of each form of the mannitol was analyzed using both single crystal XRD and Raman microscopy for direct comparison.

The hemihydrate form of mannitol was characterized using powder XRD collected on a Panalytical X'Pert PRO powder diffractometer at $25^{\circ} \mathrm{C}$ using $\mathrm{Cu} \mathrm{K} \alpha$ radiation in continuous mode with an interpolated step size of $0.02^{\circ}$ and a counting time of $20 \mathrm{~s}$ per step.

\subsection{Raman microscopy}

Raman microscopy was performed using a Jobin-Yvon Horiba LabRam HR 800 equipped with an $x-y$ motorized stage. Magnification (50×) was used, with an excitation wavelength of $514 \mathrm{~nm}$ (power $10 \mathrm{~mW}$ ), confocal hole $800 \mu \mathrm{m}$ diameter. A $1200 \mathrm{lmm}^{-1}$ diffraction grating was employed to record high resolution $\left(<4 \mathrm{~cm}^{-1}\right)$ spectra for accurate identification of differences in the spectra. A $300 \mathrm{lmm}^{-1}$ diffraction grating was used to maximize throughput while maintaining an adequate resolution of $8 \mathrm{~cm}^{-1}$. The Raman data were acquired using LabSpec software (Jobin-Yvon, Villeneuve d'Ascq, France).

\subsection{Investigation into the effect of orientation}

Because of the use of polarized laser light for excitation, Raman spectroscopic methods are sensitive to orientation of highly ordered systems. In order to investigate the dependence of the Raman signal on the orientation of the crystal axis to the path and polarization of the laser beam each of the single crystals was analyzed in three orientations. ' $z$ ' was measured with the long axis of the crystal parallel to the path of the incident beam (referred to as the ' $z$ ' axis in this paper). ' $x$ ' was measured with the long axis of the crystal perpendicular to the path of the incident beam but parallel to the polarization. Finally ' $y$ ' was measured with the long axis of the crystal perpendicular to the path of the incident beam and perpendicular to the polarization.

\subsection{Solution studies - annealing at $-30^{\circ} \mathrm{C}$}

\subsection{1. $T R M$}

In order to investigate the behavior of mannitol solutions at subambient temperatures TRM was performed using the Raman microscope described above in conjunction with a Linkam TMS 600 biological cryostage. Temperature control was provided by connection to a Linkam TMS 94 temperature controller via a Linkam LNP gas flow controller using liquid nitrogen as the gas source. 
One microliter samples of $3 \%(\mathrm{w} / \mathrm{v})$ mannitol solution were cooled at $10^{\circ} \mathrm{C} \mathrm{min}^{-1}$ to $-30^{\circ} \mathrm{C}$ before annealing for time periods varying between $5 \mathrm{~min}$ and $7 \mathrm{~h}$. This allowed direct comparison with results from the previous work. Raman maps at the edges of the frozen annealed samples were recorded at $10 \mathrm{~min}$ intervals in the $2600-3600 \mathrm{~cm}^{-1}$ spectral region over an area of $10.5 \times$ $10.5 \mu \mathrm{m}$ using a spacing of $1.5 \mu \mathrm{m}$ (the accumulation time for individual spectra was $6 \mathrm{~s}$ ) for the first $90 \mathrm{~min}$ of annealing time. The spectral region for mapping was chosen to enable simultaneous monitoring of $\mathrm{CH}$ and $\mathrm{OH}$ vibrational modes of mannitol and $\mathrm{OH}$ vibrational modes of ice.

At longer annealing times larger maps were acquired with $3 \mu \mathrm{m}$ spacing and accumulation times of $4 \mathrm{~s}$.

Two methods were employed to process the Raman data, the method chosen depending on the signal-to-noise ratio. For high signal-to-noise ratio data (averages, loadings and long annealing time data when the mannitol had concentrated at the edge) the low resolution spectra of the single crystals were used to construct a set of model reference spectra. The non-Raman background was subtracted using a four point linear algorithm, so that variation in the background would not disrupt the analysis. The linear combination of the individual polymorphic, amorphous and hemihydrate spectra that best fitted the spectrum of each sample presented was calculated in Microsoft Excel (Microsoft Corporation, Redmond, WA, USA). This Linear Combination of Elements (LCE) analysis was carried out using the region $2800-2950 \mathrm{~cm}^{-1}$ to limit the analysis to the mannitol bands and to exclude any possible interference from $\mathrm{OH}$ bands due to ice when analyzing the frozen systems. The contributions from the individual orientations within each crystalline form were combined to give a total contribution for each polymorph. The relative abundance of the various forms within the rim of the mannitol solution at extended annealing times was statistically examined using a one-way ANOVA. Individual differences between the mean values of the individual groups were statistically analyzed using Tukey's HSD test. In all cases $P<0.05$ denoted significance.

The accuracy of the linear combination method was checked by analyzing 10 single crystals from fresh preparations of each of the four crystalline forms (using the Walter-Levy and Nunes methods referenced above). The spectra for each of these crystals were recorded for $8 \mathrm{~s}$ at 20 and $2 \mathrm{~mW}$ to give good and poor signal-to-noise ratios to determine the influence of signal quality on the ability of Raman to correctly determine the polymorphic form within a sample.

Raman data with low signal-to-noise ratio for mannitol modes (early stage annealing studies, when the mannitol had not concentrated) were analyzed using Principal Component Analysis (PCA) carried out using The Unscrambler V9.6 (Camo, Trondheim, Norway). The spectra were mean centred and validated by full (leave-one-out) cross validation and the spectral regions for $\mathrm{CH}$ stretching $\left(2850-3100 \mathrm{~cm}^{-1}\right)$ and $\mathrm{OH}$ stretching $\left(3100-3600 \mathrm{~cm}^{-1}\right)$ were analyzed separately. Principal component (PC) loadings were analyzed by LCE to assign the mannitol forms contributing to each PC. As the mannitol signal was so weak in these early-stage annealing studies no baseline removal or normalization steps were carried out, as the high noise levels would cause significant, non-reproducible, distortions on the data. Furthermore, since each map is maintained at a fixed focal position, any changes in absolute intensity are of interest and these variations would be removed by normalization. This has the consequence that the first 2-3 PCs (depending on spectral range analyzed) were accounted for by non-Raman variation and were not employed in this study. Only chemically significant PCs were retained.

\subsection{Calibration}

Aqueous solutions of mannitol in the range $0-45 \%(\mathrm{w} / \mathrm{v})$ in 5\% increments were prepared and their Raman spectra were recorded for $1 \mathrm{~min}$. The non-Raman background was removed using two linear lines between 2830 and $3000 \mathrm{~cm}^{-1}$ and between 3000 and $3800 \mathrm{~cm}^{-1}$. The area of the Raman spectra between 2840 and $2990 \mathrm{~cm}^{-1}$ was divided by the area between 3100 and $3600 \mathrm{~cm}^{-1}$ and this ratio was correlated with the concentration of the mannitol in the solutions. Because of the physical processes eliminating mannitol from frozen solutions we were unable to obtain reliable calibration over a reasonable concentration range. Therefore the results refer to changes in relative mannitol concentration, which allow semi-quantitative measurements from separate Raman based experiments to be directly compared.

\section{Results and discussion}

\subsection{Single crystal analysis}

\subsubsection{Average Raman spectra and XRD}

Fig. 1 shows average Raman spectra for the different physical forms of mannitol (each spectrum of crystalline material is the average of the three orientations), each of

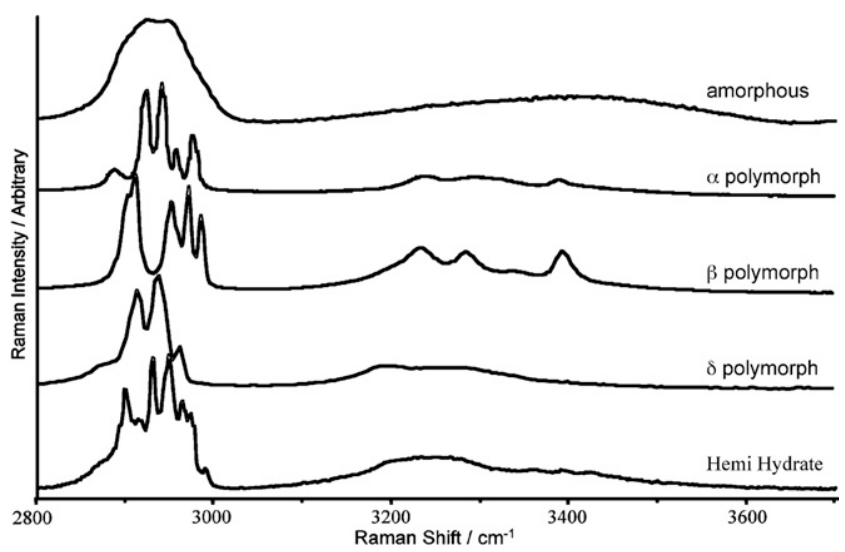

Fig. 1. Average Raman spectra in the $2800-3700 \mathrm{~cm}^{-1}$ region for amorphous mannitol, the $\alpha, \beta$ and $\gamma$ polymorphs and for the hemihydrate. 
which shows distinct band patterns allowing unambiguous classification of hydration and polymorphic form. These assignments were verified for the anhydrous forms using single-crystal XRD, which showed each polymorph to be an exact match with the literature structures determined from single crystal analyses at $100 \mathrm{~K}$ [24]. The assignments of the bands evident in the spectra are listed in Table 1. The spectra shown contain contributions from the $\mathrm{CH}$ bonds $\left(2800-3100 \mathrm{~cm}^{-1}\right)$ and from the $\mathrm{OH}$ bonds $\left(3150-3450 \mathrm{~cm}^{-1}\right)$. The $\mathrm{CH}$ bands are known to be sensitive to the chemical composition of the sample and also any forces that might affect the freedom of the bonds to vibrate, such as rotations about bonds, steric interactions and intermolecular interactions [25]. Thus, in crystalline materials the $\mathrm{CH}$ region is sensitive to both the conformation that the individual molecules adopt within the crystalline structure and also to the type of unit cell in the crystal. This latter aspect is important in the mannitol crystals as the conformation of the main backbone in individual molecules within each crystal lattice is remarkably similar and the difference between the polymorphs is predominantly that of the positioning of the $\mathrm{OH}$ hydrogen atoms, through rotation about $\mathrm{C}-\mathrm{O}$ bonds, which determines the intermolecular hydrogen bonding and thus the packing effects [24].

The $\mathrm{OH}$ region is known to be sensitive to hydrogen bonding with the literature values for mannitol $\mathrm{O}-\mathrm{H} \cdots \mathrm{O}$ ranging between 2.65 and $2.81 \AA$ (24), (26), (27), with strong hydrogen bond interactions reducing the strength of the true $\mathrm{O}-\mathrm{H}$ bond and so reducing the Raman shift. Thus it can be seen from the spectra (Fig. 1) that some of the hydrogen bonds in the $\alpha$ polymorph are stronger than the other anhydrous polymorphs, with the lowest band occurring at $3200 \mathrm{~cm}^{-1}$. Furthermore, the main band in the $\alpha$ polymorph is significantly wider than the $\beta$ or $\delta$ polymorphs, suggesting that the strength of the hydrogen bonds is much more variable and less organized for this form. The $\mathrm{OH}$ bands are similar in shape between the $\beta$ and $\delta$ polymorphs, though the intensity is shifted more towards the medium strength hydrogen bonds in the $\beta$ form.

The "hemihydrate" crystals obtained using the method of Nunes et al. [13] were revealed to be a mixture of $\beta, \delta$ forms as well as the hemihydrate when analyzed with both the Raman and XRD. This mixture of polymorphs and the hemihydrate is consistent with the synchrotron data in [19]. In the Raman data, the signal from pure hemihydrate shows two much broader $\mathrm{OH}$ bands when compared with the anhydrous forms (Fig. 1). This suggests that the water molecule has a much less restricted range of hydrogen bonding interactions than the crystalline anhydrous forms of mannitol.

\subsubsection{Orientation effects}

The data acquired in each of the three possible orientations relative to the laser are shown in Fig. 2a-d, for each of the anhydrous polymorphic forms and the hemihydrate. The largest orientation effects are observed for the $\alpha$ polymorph, with considerable changes in relative intensity of certain bands, several band shifts and a band $\left(2986 \mathrm{~cm}^{-1}\right)$ that dominates the vertical orientation of the crystal $(z)$, but is moderate and weak in the remaining two orientations. The $\beta, \delta$ forms and hemihydrate show much less dramatic changes in relative intensities of the various constituent bands, but the spectra of each orientation are nevertheless significantly different.

Table 1

Raman bands present in the spectra of each physical form of mannitol

\begin{tabular}{|c|c|c|c|c|c|c|}
\hline & Chemical bond & $\alpha$ Polymorph & $\beta$ Polymorph & $\delta$ Polymorph & Hemihydrate & Amorphous \\
\hline 2866 & $\mathrm{C}-\mathrm{H}$ & - & - & - & $\mathrm{sh}^{\mathrm{a}}$ & - \\
\hline 2885 & $\mathrm{C}-\mathrm{H}$ & $\mathrm{w}^{\mathrm{b}}$ & - & $\mathrm{w}$ & - & - \\
\hline 2898 & $\mathrm{C}-\mathrm{H}$ & - & sh & - & s & sh \\
\hline 2910 & $\mathrm{C}-\mathrm{H}$ & - & $\mathrm{s}^{\mathrm{c}}$ & s & $\mathrm{m}^{\mathrm{d}}$ & - \\
\hline 2923 & $\mathrm{C}-\mathrm{H}$ & $\mathrm{s}$ & - & - & $\mathrm{s}$ & $\mathrm{s}$ \\
\hline 2937 & $\mathrm{C}-\mathrm{H}$ & $\mathrm{s}$ & - & s & sh & - \\
\hline 2943 & $\mathrm{C}-\mathrm{H}$ & - & - & - & $\mathrm{s}$ & - \\
\hline 2950 & $\mathrm{C}-\mathrm{H}$ & - & s & - & - & s \\
\hline 2955 & $\mathrm{C}-\mathrm{H}$ & $\mathrm{m}$ & - & $\mathrm{m}$ & $\mathrm{m}$ & - \\
\hline 2968 & $\mathrm{C}-\mathrm{H}$ & $\mathrm{sh}$ & $\mathrm{s}$ & - & $\mathrm{m}$ & - \\
\hline 2976 & $\mathrm{C}-\mathrm{H}$ & $\mathrm{s}$ & - & - & - & - \\
\hline 2985 & $\mathrm{C}-\mathrm{H}$ & $\mathrm{vw}$ & s & - & $\mathrm{w}$ & - \\
\hline 3195 & $\mathrm{O}-\mathrm{H}$ & w & - & - & - & - \\
\hline 3232 & $\mathrm{O}-\mathrm{H}$ & w & $\mathrm{m}$ & - & $\mathrm{m}, \mathrm{v}$ broad & - \\
\hline 3285 & $\mathrm{O}-\mathrm{H}$ & w, broad & $\mathrm{m}$ & w, broad & - & - \\
\hline 3337 & $\mathrm{O}-\mathrm{H}$ & - & w, broad & - & - & - \\
\hline 3390 & $\mathrm{O}-\mathrm{H}$ & $\mathrm{w}$ & $\mathrm{m}$ & - & $\mathrm{w}, \mathrm{v}$ broad & $\mathrm{v}$ broad \\
\hline
\end{tabular}

${ }^{\text {a }}$ Shoulder (not fully resolved from adjoining band).

b Weak.

${ }^{\mathrm{c}}$ Strong.

${ }^{\mathrm{d}}$ Medium. 

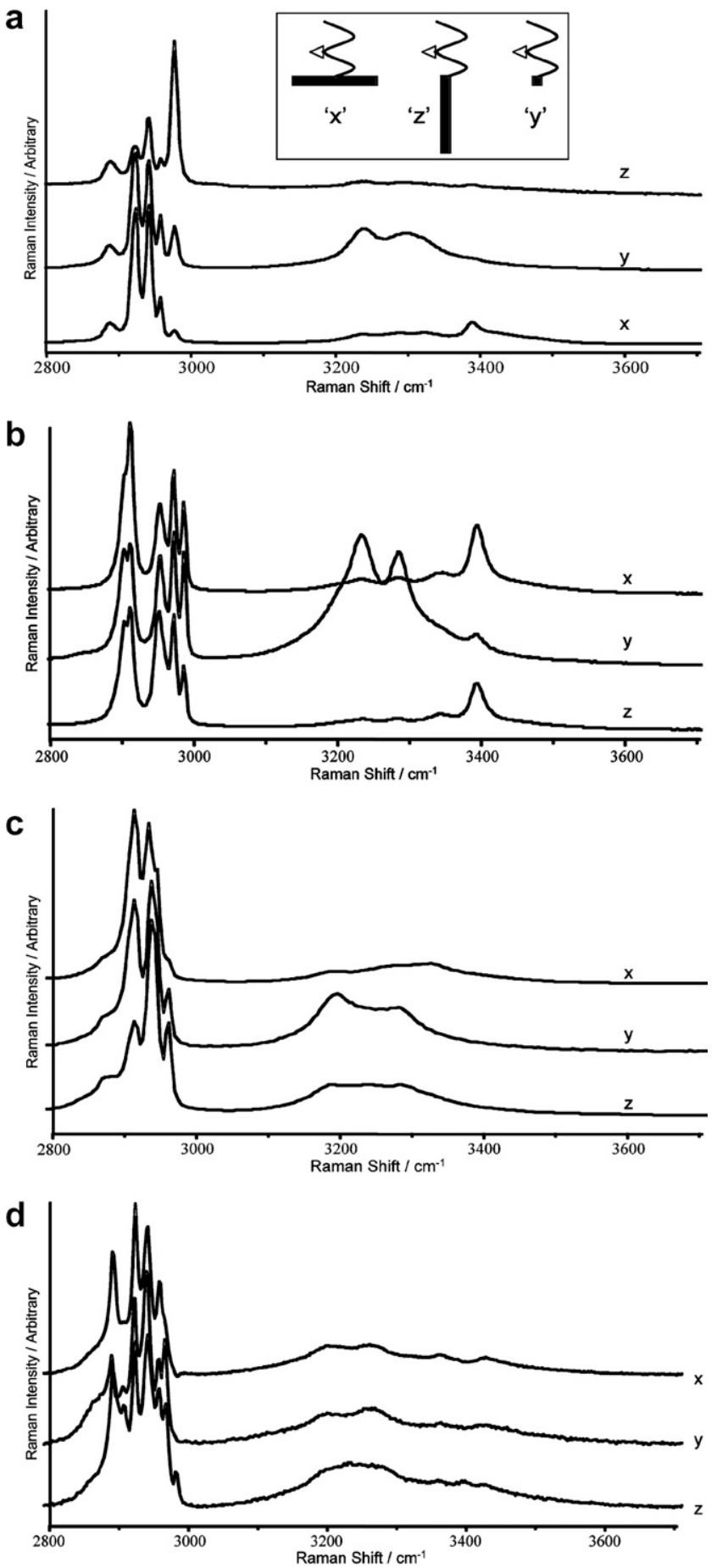

Fig. 2. Raman spectra in the $2800-3700 \mathrm{~cm}^{-1}$ region for different orientations of the crystalline forms (a) $\alpha$, (b) $\beta$, (c) $\delta$, (d) hemihydrate. Inset is a schematic diagram of the orientation of the single crystal to the path and polarization of the incident radiation.

\subsubsection{LCE method verification}

The method correctly predicted the anhydrous polymorphic form on 29 of the 30 reference anhydrous crystals and revealed a mixed polymorphic form on one other, this was attributed to the $\delta$ form transforming to the more stable $\beta$ form. On average the expected polymorph accounted for $89.4 \pm 9.3 \%$ of the Raman signal, with the remaining
$10.6 \%$ comprised of a mixture of forms. As described above, the hemihydrate preparation was revealed to be a mixture of three forms, the hemihydrate as well as the $\beta$ and $\delta$ anhydrous forms. This was also shown in the LCE analysis; a mixture of these three forms was found in the reference spectra obtained from the hemihydrate preparation. In all cases one of these three forms dominated (ca. $90 \%$ ) and no signals intermediate between these forms were observed.

These results suggest that the LCE method is useful in determining the predominant polymorphic form present in crystals of mannitol and its hemihydrate, but that the detection of minor contributors is unreliable (e.g. for results contributing to less than $10 \%$ ), due to the problem of overfitting. Use of more sensitive processing methods such as PCA would be expected to allow analysis of the trace constituents of a polymorphic sample, although LCE needs to be employed to accurately assign the polymorphic forms contributing to each factor identified.

\subsubsection{Subambient behavior of 3\% (w/v) mannitol solutions}

Previously XRD analysis had detected formation of the $\beta$ polymorph only [17]. However, in the present study a large number of replicates were performed revealing variability in the crystallization behavior of mannitol (Table 2). Furthermore, it has been shown that orientation effects can give rise to significant differences in peak intensity during XRD analysis [28]. Indeed Roberts et al. investigated the effect of orientation on XRD analysis of mannitol powders and concluded that XRD data obtained from mannitol samples are particularly prone to orientation effects [29]. This is also the case for Raman microscopy, but the changes in the Raman signal induced by orientation are significantly less than those induced by polymorphic change, as demonstrated in Fig. 2.

Ultimately it is desirable to be able to quantify the concentration of mannitol in each location, rather than simply qualitatively detecting its presence. In the aqueous solutions the ratio of the area of the mannitol derived Raman band in the $\mathrm{CH}$ stretching region $\left(2800-3100 \mathrm{~cm}^{-1}\right)$ to the area of the full spectrum is very well correlated $\left(R^{2}=0.999\right)$ with concentration of mannitol in liquid aqueous solutions between $1 \% \mathrm{w} / \mathrm{v}$ and $45 \% \mathrm{w} / \mathrm{v}$. Unfortunately the behavior of sub-ambient mannitol solutions presents

Table 2

The average proportion of the total spectral intensity (\%) of mannitol forms present in the rim of a drop of frozen 3\% mannitol solution at 90 min annealing time

\begin{tabular}{llc}
\hline Morphology & Mean $(\%)$ & Standard deviation \\
\hline$\alpha$ & 16.7 & 5.4 \\
$\beta$ & 37.2 & 11.0 \\
$\delta$ & 22.2 & 8.8 \\
Hemihydrate & 20.6 & 5.4 \\
Amorphous & 3.4 & 3.3
\end{tabular}

Determined by linear combination (LCE) of the reference spectra for each of the pure polymorphic samples $(n=6)$. 
severe problems for the calibration of mannitol concentration in ice as the more concentrated a solution the more rapidly the mannitol is expelled from the drop upon freezing (data not shown). Since the Raman effect occurs predominantly at the surface, when recording opaque samples it is not possible to simultaneously measure the outer crystalline mannitol and inner mannitol/ice solution with a reasonable degree of confidence. Furthermore, the efficiency of the Raman scattering can vary between both polymorphic form and orientation. The addition of any internal standard to either the crystalline mannitol or the aqueous mannitol is likely to disrupt the system under investigation, rendering it unsuitable. For these reasons discussion below is restricted to changes in relative mannitol concentration, which allow semi-quantitative measurements from separate Raman, based experiments to be directly compared.

\subsubsection{Effect of annealing at $-30^{\circ} \mathrm{C}$}

Of most interest in terms of the behavior of mannitol during freeze-drying is the initial period up to around 90 min annealing at $-30{ }^{\circ} \mathrm{C}$ since this is typical of an annealing step included in a pharmaceutical freeze-drying cycle. During this period both the mannitol and ice structures were observed to change subtly and so the two regions of the Raman spectrum relating to these components were analyzed separately. The signal to noise level of the spectra was much lower than that used in the analysis of the single crystals detailed above, primarily due to the significantly lower density of the mannitol in a $3 \%$ (w/v) solution. This prevented reliable use of LCE to determine the form of mannitol present in the sample. Thus PCA was used to give more sensitive measurement of the variation in the mannitol signal present.

The loadings from PCA of the region from 2800 to $3100 \mathrm{~cm}^{-1}$ that contained contributions from the mannitol

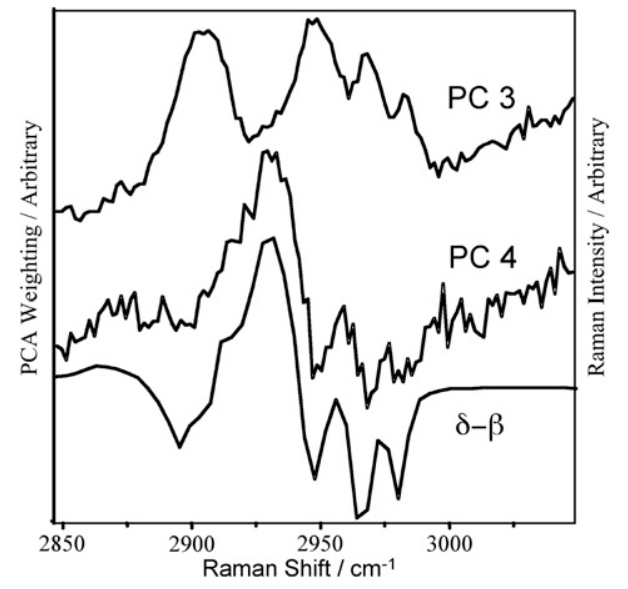

Fig. 3. PCA loadings related to changes in the Raman signal of mannitol (2850-3100 $\mathrm{cm}^{-1}$ ) over the first $90 \mathrm{~min}$ of annealing at $-30^{\circ} \mathrm{C}$ for a $3 \% \mathrm{w} /$ $\mathrm{v}$ mannitol solution. For comparison the result of subtracting the Raman spectra of $\beta$ crystalline mannitol from the spectrum of $\delta$ mannitol is also included (for each the spectrum is the average of all orientations). bands are compared with the result of subtracting the spectrum of $\beta$ mannitol from that of $\delta$ mannitol in Fig. 3. The loadings for PC3 match the pattern of intensities that would be expected from a mixture of $\beta$ and $\delta$ polymorphs and can therefore be related to the total mannitol concentration. The loadings from each PC were investigated using LCE as described above for the polymorphic forms, which confirmed that the only significant contributors to PC3 were $\beta$ and $\delta$ polymorphs. The amorphous, $\alpha$ polymorph and the hemihydrate forms were not detected in significant amounts in these early-stage annealing samples. The negative bands of PC4 match the positions of $\beta$ mannitol, while the positive bands match the positions typical of $\delta$ mannitol. Thus each data point with a high score represents a spectrum with a higher amount of $\delta$ mannitol while a low score represents samples where the $\beta$ form predominates. Fig. 4 presents the effect of annealing on analyses at the centre and at the edge of the frozen drop in terms of PC3 (total mannitol) and $4(\delta$ vs. $\beta$ ) scores plotted from this PCA for the centre and the edge of the frozen drop compared with the results of the previously reported DSC analysis.

The PC3 score for analyses from the edge of the frozen drop shown in Fig. 4 indicates that the mannitol crystallizes rapidly (by time of initial measurement, $<1 \mathrm{~min}$ ) and that the proportion of mannitol at the edge of the frozen drop increases throughout the annealing process, with a greater rate of increase after $\sim 35 \mathrm{~min}$. This suggests that

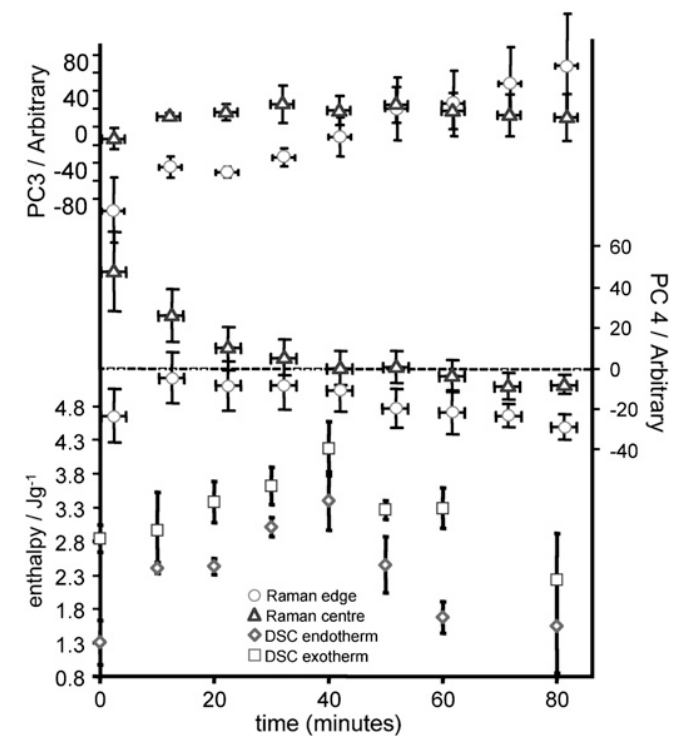

Fig. 4. Variation in mannitol signal $\left(2850-3100 \mathrm{~cm}^{-1}\right)$ as measured by TRM/PCA in the centre of a frozen sample of 3\% mannitol solution compared with DSC data acquired over the same time period [1]. The PCA scores from the Raman data are plotted for the edge ( $\circ$ ) and centre $(\triangle)$ of the frozen drop with PC4 corresponding to a higher proportion of $\delta$ form, while a negative score corresponds to a higher proportion of $\beta$ mannitol. PC3 is positively correlated with the total mannitol signal. $y$ error bars indicate standard deviation, $x$ error bars indicate the range of times over which the data were collected. The DSC data are displayed as enthalpy of endotherm $(\diamond)$ and enthalpy of exotherm $(\square)$. Error bars indicate standard deviation. 
the rim of crystalline mannitol is developing from the earliest stages, even before it is evident visually. While the trend in the PC4 scores from the centre of the drop indicates that the ratio of $\delta$ to $\beta$ decreased rapidly during the initial stage of annealing (up to $\sim 35 \mathrm{~min}$ ) and thereafter continued to decrease, albeit at a lower rate. At the edge a similar decline in the relative amount of the $\delta$ form was also seen at longer annealing times suggesting that the $\beta$ form is preferred under the conditions of the experiment. These results help to explain the apparent two stage process revealed in the previously reported DSC data. They indicate that the $\delta$ mannitol present in the system converts to the $\beta$ form. The endotherm observed in the DSC data corresponds to that of an endothermic relaxation accompanying a glass transition observed after cooling and rewarming the annealed samples, while the exotherm is due to this amorphous material crystallizing into the $\beta$ form. Therefore at annealing times longer than $\sim 35$ min the amount of the $\delta$ form able to undergo the process observed in the DSC experiments decreases since the stable $\beta$ polymorph has already been formed during annealing.

The $\mathrm{O}-\mathrm{H}$ stretching bands from the ice were also investigated as it is well established that these bands are sensitive to the ice lattice structure [27]. Their positions are correlated with parameters such as bond length of the donor $\mathrm{O}-\mathrm{H}$ bond involved in hydrogen bonding, which is inversely related to hydrogen bonding strength [26]. Fig. 5 shows the most relevant PCs from PCA of the $\mathrm{OH}$ stretching modes. (Higher PCs were found to account for variations in the non-Raman background.) It is clear that there is more than one process occurring within the ice lattice during the annealing period as the changes in hydrogen bonding require two PCs to fully account for them. PC4 represents a decrease at $\sim 3125 \mathrm{~cm}^{-1}$ against an increase

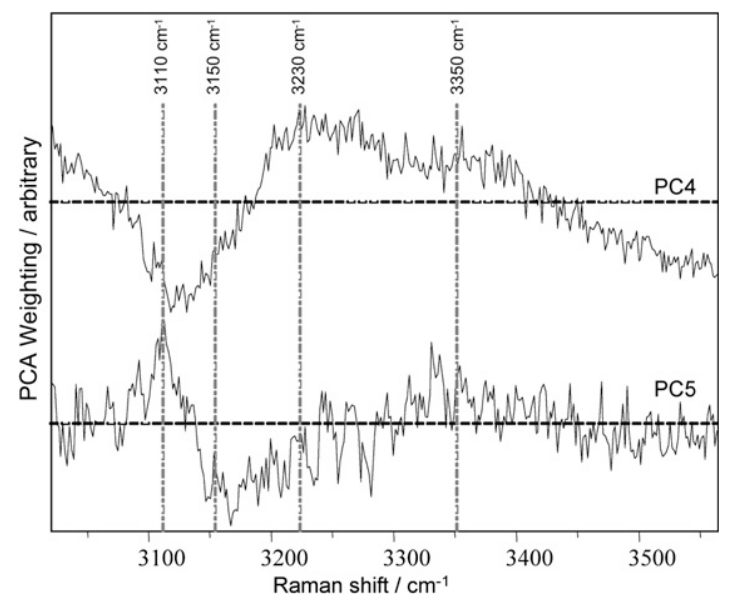

Fig. 5. PCA loadings from the ice bands obtained from Raman data between 3000 and $3600 \mathrm{~cm}^{-1}$, attributable to $\mathrm{O}-\mathrm{H}$ stretching vibrations, recorded over the first $90 \mathrm{~min}$ of annealing at $-30{ }^{\circ} \mathrm{C}$ for a $3 \% \mathrm{w} / \mathrm{v}$ mannitol solution. The Raman shifts of the ice bands are known to be related to the strength of the hydrogen bonding within the ice lattice with a lower shift (left hand side) corresponding to a stronger hydrogen bond. Highlighted Raman shifts are discussed in the text. from 3200 to $3400 \mathrm{~cm}^{-1}$. Using the correlation between the $\mathrm{O}-\mathrm{H}$ stretching frequency and donor bond length [30] we can determine that a higher score represents weak and medium hydrogen bonds (with donor $\mathrm{O}-\mathrm{H}$ bond lengths estimated at $97.3-99.3 \mathrm{pm}$ ) as the loadings have positive bands at higher Raman shifts while a low score is related to strong hydrogen bonds $(99.9 \mathrm{pm})$. PC5 measures the ratio of intensity at 3110 to $3160 \mathrm{~cm}^{-1}$, which is the ratio of very strong hydrogen bonds to medium-strong hydrogen bonds (estimated donor bond lengths 100.1 and $99.6 \mathrm{pm}$ ). A high score for PC5 is indicative of strong hydrogen bonding, while a low score is indicative of medium strength hydrogen bonding.

Fig. 6 shows the variation in the resultant scores. The plot of PC4 scores with annealing time shows no systematic variation in the centre of the drop. However, at the edge there is a more reproducible pattern of change (except the first, highly variable data point) with a clear weakening of the hydrogen bonding over time. PC5 shows a distinct downward trend with time similar to that observed in the polymorphic transition (PC4) for the centre of the frozen drop, as shown in Fig. 4, but with a time lag between these two variations. These data show the reorganization of the ice surrounding the mannitol consequent upon the major restructuring caused by the $\delta$ to $\beta$ transition of the mannitol. This again helps to explain the findings of the previous DSC experiments since it indicates a lag between the process in the centre and at the edge of the system corresponding to the disappearance of the $\delta$ form in the centre and the appearance of the $\beta$ form at the edge.

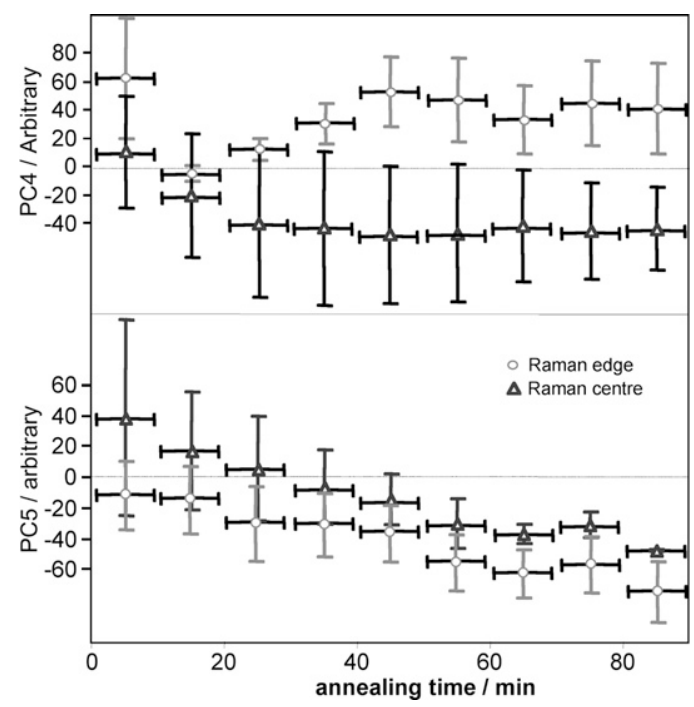

Fig. 6. The PCA scores (calculated from loadings in Fig. 5) from the Raman data between 3000 and $3600 \mathrm{~cm}^{-1}$, attributable to $\mathrm{O}-\mathrm{H}$ stretching vibrations, against annealing time at $-30{ }^{\circ} \mathrm{C}$ depicting the main changes occurring in the Raman signal attributable to ice hydrogen bonding. The standard deviation is shown by the $y$ error bars, while the range of times over which the data were collected is indicated by the $x$ error bars. 


\subsubsection{Raman maps}

Raman maps allowed the visualization of the effect of location on the total mannitol concentration and the polymorphic form. The first of these, presented in Fig. 7, illustrates the movement of mannitol in the initial period of annealing, before the rim was observable optically. There is a clear accumulation of mannitol at the edge of the drop with increasing annealing time. In the centre the behavior was more variable, as would be expected from diffusion of the mannitol within this region. In both regions a mixture of $\beta$ and $\delta$ polymorphs was observed.

Fig. 8 shows the effect on the system of annealing for extended periods of time where the rim is well developed and the Raman maps confirmed the presence of a boundary region that was rich in crystalline mannitol and low in ice. Both the concentration of mannitol and the extent of the boundary at the edge of the sample increased with annealing time while the main body of the drop receded.
Light microscopy before and after mapping confirmed the band growth (Fig. 9).

In order to identify the forms of mannitol present in this extended annealing dataset LCE was employed as the data showed a sufficiently high signal to noise ratio. The results of the LCE analysis of the Raman data for frozen 3\% mannitol solutions (Table 2) show that the mannitol preferentially crystallizes, with very little amorphous content. All of the anhydrous polymorphic forms and the hemihydrate occurred in the frozen drop with the $\beta$ polymorph the dominant crystalline form.

Statistical analysis showed that in all samples the differences in the relative proportion of amorphous compared to $\beta$ form were extremely significant $(P<0.001)$, as was the amount of $\beta$ to $\alpha$. The difference between amounts of amorphous and hemihydrate was very significant $(P<0.01)$ as was the difference between amounts of amorphous and $\partial$ and between $\beta$ and hemihydrate. The differ-

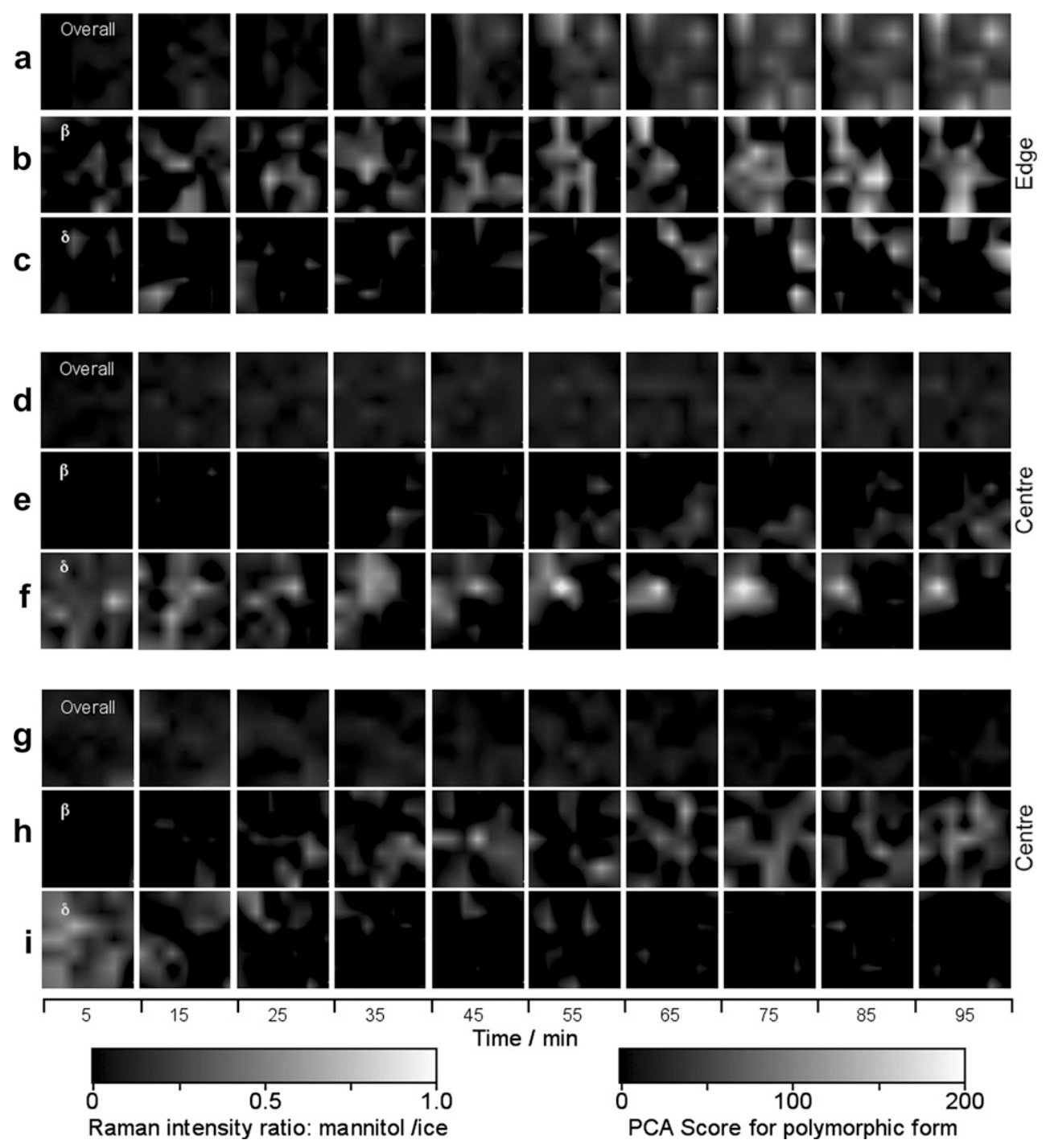

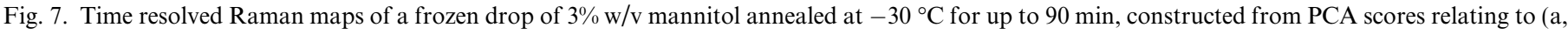

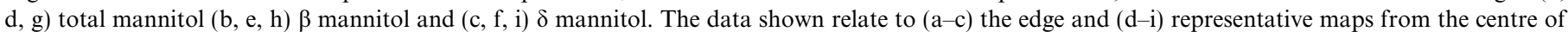
two independent samples exhibiting different behavior in terms of variation in total mannitol. 


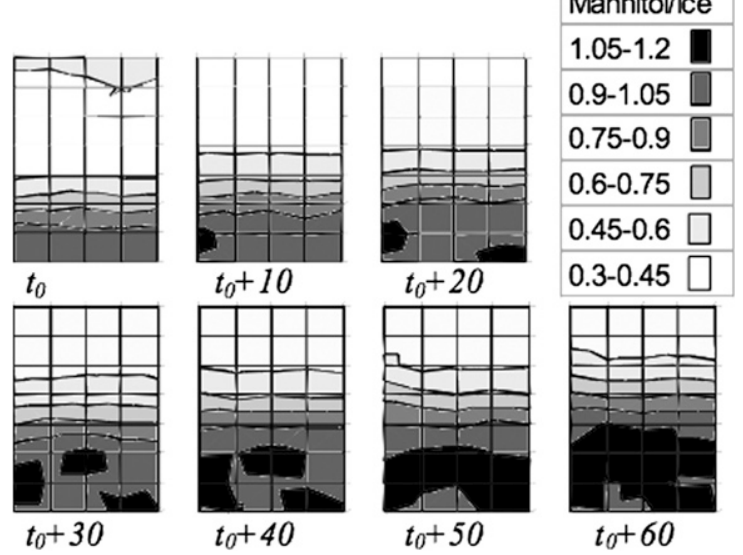

Fig. 8. Raman maps of the distribution of mannitol within a $3 \% \mathrm{w} / \mathrm{v}$ mannitol solution annealed at $-30{ }^{\circ} \mathrm{C}$ constructed using LCE. The ratio mapped is the ratio of the spectral intensity due to mannitol vs. the spectral intensity due to water. Initial imaging acquired at $t_{0}=150 \mathrm{~min}$. Individual squares on maps indicate a $3 \mu \mathrm{m} \times 3 \mu \mathrm{m}$ area.

a
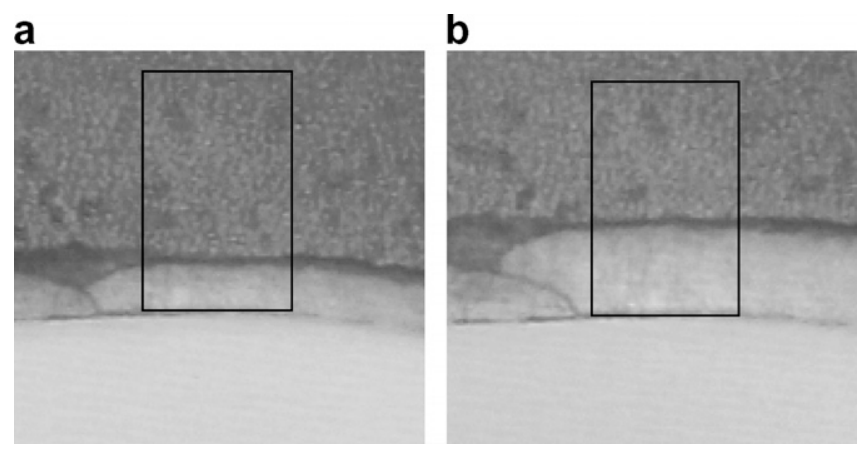

Fig. 9. Optical microscopy image showing the area of the drop featured in Fig. 7 (a) prior to mapping (b) after mapping. The area is indicated by the rectangle.

ence between relative proportions of $\alpha$ and amorphous was significant as was the difference between the proportions of $\beta$ and $\partial$ forms. There was no significant difference between the relative proportions of $\alpha$ versus $\partial, \alpha$ versus hemihydrate or $\partial$ versus hemihydrate.

This finding has implications for the stability of mannitol-containing formulations. For example, the results show that it is likely that the polymorphic form dominating in the freezing/annealing step will not be the same polymorph that dominates at the end of the process. There will certainly be an effect of other excipients and protein on the crystallization behavior, as discussed in the introduction.

\section{Conclusion}

We have demonstrated that Raman microscopy can be used to determine the polymorphic form of mannitol, and have used this capability in a TRM setting to map the effect of annealing on the crystallization behavior of this material at different locations with a frozen sam- ple. The spatial resolution of the technique has allowed targeted analysis of the sample revealing local variations in behavior indicating that the mannitol is crystallized rapidly $(<1 \mathrm{~min})$. The events observed using DSC were attributed to transitions between polymorphic form and the subsequent reorganizing of the ice surrounding the mannitol, which were most significant during the first $\sim 35$ and $\sim 65 \mathrm{~min}$ of annealing, respectively. The excluded phase that had been noted previously contains a much higher concentration of mannitol than the centre of the drop and this concentration increases with annealing. Furthermore, the method has demonstrated that both the $\beta$ and $\delta$ forms can appear upon annealing, with no significant preference for either form initially. However, the $\beta$ form dominates after the annealing conditions described here, both in the centre and at the edge of the drop. This, coupled with the ability of the technique to visualize different regions within a structure, has shed new light on previous DSC, CSM and TXRD data and allowed the clear location of the crystallizing material within this sample for the first time. It clearly has potential for further use in following crystallization processes particularly in frozen systems.

\section{Acknowledgments}

The Centre for Clinical Raman Microscopy acknowledges the financial support of the Northern Ireland Health and Personal Social Services Research and Development Office (Grant No. SPI/2384/03). Purchase of the Raman microscope was assisted by funding from BBSRC (JREI Grant No. 18471). VK thanks Professor David Jones for help with the statistical analysis.

\section{References}

[1] A.J. Cannon, E.H. Trappler, The influence of lyophilization on the polymorphic behavior of mannitol, PDA J. Pharm. Sci. Technol. 54 (2000) 13-22.

[2] K.-I. Izutsu, S. Yoshioka, T. Terao, Effect of mannitol crystallinity on the stabilization of enzymes during freeze-drying, Chem. Pharm. Bull. 42 (1994) 5-8.

[3] K.-I. Izutsu, S. Yoshioka, T. Terao, Decreased protein stabilizing effects of cryoprotectants due to crystallization, Pharm. Res. 10 (1993) $1232-1237$.

[4] R.E. Johnson, C.F. Kirchhoff, H.T. Gaud, Mannitol-sucrose mixtures - versatile formulations for protein lyophilization, J. Pharm. Sci. 91 (2002) 914-922.

[5] A.I. Kim, M.J. Akers, S.L. Nail, The physical state of mannitol after freeze-drying: effects of mannitol concentration, freezing rate and a noncrystallising cosolute, J. Pharm. Sci. 87 (1998) 931-935.

[6] A. Pyne, K. Chatterjee, R. Suryanarayanan, Solute crystallization in mannitol-glycine systems - implications on protein stabilization in freeze-dried formulations, J. Pharm. Sci. 92 (2003) 2272-2283.

[7] S. Passot, F. Fonseca, M. Alarcon-Lorca, D. Rolland, M. Marin, Physical characterisation of formulations for the development of two stable freeze-dried proteins during both dried and liquid storage, Eur. J. Pharm. Biopharm. 60 (2005) 335-348.

[8] A. Pyne, R. Surana, R. Suryanarayanan, Crystallization of mannitol below $\mathrm{Tg}$, during freeze-drying in binary and ternary aqueous systems, Pharm. Res. 19 (2002) 901-908. 
[9] C. Telang, L. Yu, R. Suryanarayanan, Effective inhibition of mannitol crystallization in frozen solutions by sodium chloride, Pharm. Res. 20 (2003) 660-667.

[10] R.K. Cavaturand, R. Suryanarayanan, Characterization of frozen aqueous solutions by low temperature X-ray powder diffractometry, Pharm. Res. 15 (1998) 94-199.

[11] A. Burger, J.O. Henck, S. Hetz, J.M. Rollinger, A.A. Weissnicht, H. Stottner, Energy/temperature diagram and compression behavior of the polymorphs of D-mannitol, J. Pharm. Sci. 89 (2000) 457-468.

[12] R. Haikala, R. Eerola, V.P. Tanninen, J. Yliruusi, Polymorphic changes of mannitol during freeze-drying: effect of surface-active agents, PDA J. Pharm. Sci. Technol. 51 (1997) 96-100.

[13] C. Nunes, R. Suryanarayanan, C.E. Botez, P.W. Stephens, Characterization and crystal structure of D-mannitol hemihydrate, J. Pharm. Sci. 93 (2004) 2800-2809.

[14] N.A. Williams, Y. Lee, G.P. Polli, T.A. Jennings, The effects of cooling rate on phase transitions and associated vial breakage in frozen mannitol solutions, J. Parenter. Sci. Technol. 40 (1986) 135-141.

[15] J.F. Carpenter, M.J. Pikal, B.S. Chang, T.W. Randolph, Review article; rational design of stable lyophilized protein formulations: some practical advice, Pharm. Res. 14 (1997) 969-975.

[16] X.F. Luand, M.J. Pikal, Freeze-drying of mannitol-trehalose-sodium chloride-based formulations: the impact of annealing on dry layer resistance to mass transfer and cake structure, Pharm. Dev. Technol. 9 (2004) 85-95.

[17] V.L. Kett, S. Fitzpatrick, B. Cooper, D.Q.M. Craig, An investigation into the subambient behavior of aqueous mannitol solutions using differential scanning calorimetry, cold stage microscopy, and X-ray diffractometry, J. Pharm. Sci. 92 (2003) 1919-1929.

[18] C.V. Raman, A new radiation, Indian J. Phys. 2 (1928) 387.

[19] C. Telang, R. Suryanarayanan, L. Yu, Crystallization of D-mannitol in binary mixtures with $\mathrm{NaCl}$ : phase diagram and polymorphism, Pharm. Res. 20 (2003) 1939-1945.
[20] J.J. Andrew, M.A. Browne, I.E. Clark, T.M. Hancewicz, A.J. Millichope, Raman imaging of emulsion systems, Appl. Spectrosc. 52 (1998) 790-796.

[21] L. Zhang, M.J. Henson, S.S. Sekulic, Multivariate data analysis for Raman imaging of a model pharmaceutical tablet, Anal. Chim. Acta 545 (2005) 262-278.

[22] S. Sasic, D.A. Clark, J.C. Mitchell, M.J. Snowden, Raman line mapping as a fast method for analyzing pharmaceutical bead formulations, Analyst 130 (2005) 1530-1536.

[23] L. Walter-Levy, Cristallochimie - Sur les Varieties Cristallines du Dmannitol, C. R. Acad. Sci. Paris C 267 (1968) 1779-1782.

[24] F.R. Fronczek, H.N. Kamel, M. Slattery, Three polymorphs $(\alpha, \beta$ and $\delta$ ) of D-mannitol at $100 \mathrm{~K}$, Acta Crystallogr. C59 (2003) 567570.

[25] P. Tandon, Phase transitions in oleic acid as studied by X-ray diffraction and FT-Raman spectroscopy, J. Mol. Struct. 524 (2000) 201-215.

[26] H.D. Lutzand, C. Jung, Water molecules and hydroxide ions in condensed materials; correlation of spectroscopic and structural data, J. Mol. Struct. 404 (1997) 63-66.

[27] P. Pruzan, Phase diagram of ice in the VII-VIII-X domain. Vibrational and structural data for strongly compressed ice VIII, J. Raman Spectrosc. 34 (2003) 591-610.

[28] V.J. Hurst, P.A. Schroeder, R.W. Styron, Accurate quantification of quartz and other phases by powder X-ray diffractometry, Anal. Chim. Acta 337 (1997) 233-252.

[29] S.N.C. Roberts, A.C. Williams, I.M. Grimsey, S.W. Booth, Quantitative analysis of mannitol polymorphs. X-ray powder diffractometry - exploring preferred orientation effects, J. Pharm. Biomed. Anal. 28 (2002) 1149-1159.

[30] A.G. Csaszar, G. Czako, T. Furtenbacher, J. Tennyson, V. Szalay, S.V. Shirin, N.F. Zobov, O.L. Polyansky, On equilibrium structures of the water molecule, J. Chem. Phys. 122 (2005). 